\title{
Dynamical models for dissipative localized waves of the complex Ginzburg-Landau equation
}

\author{
Eduard N. Tsoy \\ Physical-Technical Institute of the Uzbek Academy of Sciences, Mavlyanov street, 2-B, Tashkent, 700084, Uzbekistan \\ Adrian Ankiewicz and Nail Akhmediev \\ Optical Sciences Group, Research School of Physical Sciences and Engineering, The Australian National University, \\ Canberra, Australian Capital Territory 0200, Australia \\ (Received 2 November 2005; published 29 March 2006)
}

\begin{abstract}
Finite-dimensional dynamical models for solitons of the cubic-quintic complex Ginzburg-Landau equation (CGLE) are derived. The models describe the evolution of the pulse parameters, such as the maximum amplitude, pulse width, and chirp. A clear correspondence between attractors of the finite-dimensional dynamical systems and localized waves of the continuous dissipative system is demonstrated. It is shown that stationary solitons of the CGLE correspond to fixed points, while pulsating solitons are associated with stable limit cycles. The models show that a transformation from a stationary soliton to a pulsating soliton is the result of a Hopf bifurcation in the reduced dynamical system. The appearance of moving fronts (kinks) in the CGLE is related to the loss of stability of the limit cycles. Bifurcation boundaries and pulse behavior in the regions between the boundaries, for a wide range of system parameters, are found from analysis of the reduced dynamical models. We also provide a comparison between various models and their correspondence to the exact results.
\end{abstract}

DOI: 10.1103/PhysRevE.73.036621

PACS number(s): 05.45.Yv, 04.30.Nk, 42.65.Sf, 42.65.Tg

\section{INTRODUCTION}

Distributed dissipative systems demonstrate complex spatiotemporal dynamics. The interplay between the energy pump, losses, nonlinearity, dispersion, and other effects results in a rich variety of structures [1-4]. A dissipative soliton (DS) and a front (kink) are two basic examples of such structures [1,2]. A DS is a localized wave with a pulselike profile for its field. A front represents a structure with a sharp transition between two plane (or continuous) waves with different amplitudes. The parameters of DSs and fronts depend mainly on the system parameters, so different initial conditions often converge to the same localized state. Recently, a new type of localized dissipative wave, namely, the class of pulsating solitons, was found in numerical simulations [5-7] and was then observed in experiments with soliton lasers [8]. Pulsating solitons (PSs) are characterized by a (quasi) periodic variation of their parameters [9], such as a pulse width and amplitude. PSs can also be called "dissipative breathers."

The complex Ginzburg-Landau equation (CGLE) is one of the universal models used in describing dissipative systems. Examples of its application include pulse generation by passively mode-locked soliton lasers [10], signal transmission in all-optical communication lines [11], traveling waves in binary fluid mixtures [12], as well as pattern formation in many other physical systems [2]. Depending on the system parameters, the CGLE has different types of solutions, including solitons, fronts [4], and pulsating solitons [5-9].

Although exact solutions of the CGLE do exist [4], they can be presented explicitly only for certain relations between the parameters of the equation. Furthermore, so far, only stationary solutions of the CGLE are known in analytical form. Since the CGLE is characterized by several parameters, it is difficult to find a correspondence between different regions in the parameter space and various types of localized waves. The solution to this problem usually requires massive numerical simulations with different sets of parameters and initial conditions [6]. This extensive work can be avoided if we are able to find simplified models for the existence of localized solutions. As a first step in this direction, in the present work, we propose a reduction of the cubic-quintic CGLE to finite-dimensional dynamical models. As a result of the reduction, we arrive at models which provide approximate expressions for localized solutions of the CGLE. We also show that bifurcations in the models are related to corresponding transformations between the CGLE solutions.

The reduced models are obtained by applying the method of moments, developed by Maimistov [13] for the modified nonlinear Schrödinger equation (NLSE). The method suggests a way of dealing with the system by using some integral characteristics of the field under consideration. Following this approach, one can obtain a dynamical system for the evolution of several variables that correspond to the soliton parameters, such as the pulse amplitude and width. In principle, there is an infinite number of equations for the moments. Using the complete set of these equations, one can obtain exact results. However, in practice, one uses a trial function with a finite number of parameters, and this is the way to obtain a significant reduction in the number of variables used in the problem. The number of parameters in the trial function should correspond to the number of moments.

The paper is organized as follows. In Sec. II, we introduce the CGLE and discuss a lower estimate for the cubic gain in the system using plane wave solutions. In Sec. III, we briefly outline the method of moments [13]. A justification of the trial functions and the derivation of the reduced models are presented in Sec. IV. Analysis of the models is described in 
Secs. V and VI. We find boundaries in the parameter space which separate different classes of localized solutions. Section VII summarizes our results. The dynamical equations for the general form of the trial function are presented in the Appendix. The derivation of the parameters included in the one of the models (see Sec. IV B) is also discussed there. Preliminary results of the work have been published in Ref. [14].

\section{THE COMPLEX GINZBURG-LANDAU EQUATION}

The cubic-quintic complex Ginzburg-Landau equation is written as [4]

$$
\begin{aligned}
i \psi_{t}+\frac{D}{2} \psi_{x x}+\gamma|\psi|^{2} \psi= & -\nu|\psi|^{4} \psi+i \delta \psi+i \epsilon|\psi|^{2} \psi+i \beta \psi_{x x} \\
& +i \mu|\psi|^{4} \psi \equiv R[\psi],
\end{aligned}
$$

where $\psi(x, t)$ is the normalized envelope of the field, $t$ and $x$ are evolutional and spatial variables, respectively, $D$ is the dispersion coefficient, $\gamma$ and $\nu$ are the parameters of the cubic and quintic nonlinearities, respectively, $\delta$ represents the linear losses, $\epsilon$ is the nonlinear gain coefficient, $\beta$ stands for spectral filtering, and $\mu$ characterizes the saturation of the nonlinear gain. As with the nonlinear Schrödinger equation, two coefficients in (1) can be scaled away. Thus, without loss of generality, one can take $D= \pm 1$ and $\gamma= \pm 1$. Since Eq. (1) is invariant with respect to the transformation $\psi \rightarrow \psi^{\star}, D \rightarrow$ $-D, \gamma \rightarrow-\gamma$, and $\nu \rightarrow-\nu$, one can consider just two cases, viz., $D=\gamma=1$ and $D=-\gamma=-1$. In the present paper we consider the case of the same signs of $D$ and $\gamma$. The case of the opposite signs of these parameters is discussed briefly in Sec. VI.

Let us find rough lower estimates for the parameter $\epsilon$ for the localized solutions to exist. It is known that the existence of localized solutions is closely related to the existence of continuous waves or plane waves [15]. Thus, for an estimate, we look for a plane wave solution in the form $\psi$ $=u \exp [i(k x-\omega t)]$, where $u, k$, and $\omega$ are real parameters. Substituting this form into Eq. (1), one can find the following equations for the amplitude $u$ and frequency $\omega$ :

$$
\begin{gathered}
u\left[\mu u^{4}+\epsilon u^{2}+\left(\delta-\beta k^{2}\right)\right]=0, \\
\omega=\frac{D}{2} k^{2}-u^{2}\left(\gamma+\nu u^{2}\right) .
\end{gathered}
$$

Equation (2) defines the amplitude of the wave and can be easily solved:

$$
u_{1}=0, \quad u_{2-5}^{2}=-\frac{\epsilon}{2 \mu} \pm\left[\left(\frac{\epsilon}{2 \mu}\right)^{2}-\frac{\left(\delta-\beta k^{2}\right)}{\mu}\right]^{1 / 2} .
$$

We take $\delta<0$ in order to ensure that the trivial state $u=u_{1}$ is stable. Then $u_{2-5}$ are real if $\epsilon \mu<0$ and $\left(\delta-\beta k^{2}\right) \mu>0$. Therefore, a stable plane wave with arbitrary $k$ can exist if $\delta, \mu$ $<0$ and $\beta, \epsilon>0$. In the rest of the paper, we consider this category only. An additional requirement for all $u_{2-5}$ to be real is that

$$
\epsilon>2 \sqrt{\mu\left(\delta-\beta k^{2}\right)} .
$$

This condition defines the lowest value of the cubic gain $\epsilon$ that is needed in order to generate a wave with a wave number $k$.

It is clear that $u_{4}=-u_{2}$ and $u_{5}=-u_{3}$, and so we only need to consider positive values for the amplitudes. We label the solution in Eq. (4) such that $u_{3}>u_{2}>u_{1}=0$. Then the trivial solution $u=u_{1}=0$ and plane wave with amplitude $u=u_{3}$ are stable against small variations of the wave amplitude (see also [15]). Equation (3), with $u=u_{3}$, is the dispersion relation for the plane wave with the higher amplitude. The stability of plane waves with zero and the higher amplitude $u_{3}$ also means that localized solutions may exist for the same set of system parameters. The lowest value of $\epsilon$ needed for existence of localized solutions does not differ much from the one defined by (5). The existence of localized states with zero asymptotes is due to the presence of the quintic nonlinearity, namely the saturation of the gain, $\mu$, which is negative. Such states do not exist in the CGLE with the cubic nonlinearity only. All the terms in Eq. (1) play significant roles relating to the existence of pulsating solitons.

\section{THE METHOD OF MOMENTS}

In this section, we briefly outline the approach that we use to derive the dynamical model. The method of moments [13] is a reduction of the complete evolution problem with an infinite number degrees of freedom to the evolution of a finite set of pulse characteristics. For a localized solution with a single maximum, these characteristics include the peak amplitude, pulse width, center-of-mass position, and phase parameters. For an arbitrary localized field, one can introduce two integrals, namely, the energy $Q$ and momentum $P$

$$
Q=\int_{-\infty}^{\infty}|\psi|^{2} d x, \quad P=\frac{1}{2} \int_{-\infty}^{\infty}\left(\psi \psi_{x}^{*}-\psi^{*} \psi_{x}\right) d x,
$$

and higher-order generalized moments [13]

$$
\begin{gathered}
I_{1}=\int_{-\infty}^{\infty} x|\psi|^{2} d x, \quad I_{2}=\int_{-\infty}^{\infty}\left(x-x_{0}\right)^{2}|\psi|^{2} d x, \\
I_{3}=\int_{-\infty}^{\infty}\left(x-x_{0}\right)\left(\psi^{*} \psi_{x}-\psi \psi_{x}^{*}\right) d x .
\end{gathered}
$$

If the intensity $|\psi|^{2}$ is symmetric, as we consider in this paper, then $x_{0}(t)=I_{1} / Q$. The number of higher-order generalized moments is infinite. Depending on a complexity of the reduced model, we can restrict ourselves to a finite number of them. Using the original equation (1), we can derive the evolution equations for the generalized moments [13]:

$$
\begin{gathered}
\frac{d Q}{d t}=i \int_{-\infty}^{\infty}\left(\psi R^{*}-\psi^{*} R\right) d x, \\
\frac{d P}{d t}=-i \int_{-\infty}^{\infty}\left(\psi_{x} R^{*}+\psi_{x}^{*} R\right) d x,
\end{gathered}
$$




$$
\begin{gathered}
\frac{d I_{1}}{d t}=i D P+i \int_{-\infty}^{\infty} x\left(\psi R^{*}-\psi^{*} R\right) d x \\
\frac{d I_{2}}{d t}=-i D I_{3}+i \int_{-\infty}^{\infty}\left(x-x_{0}\right)^{2}\left(\psi R^{*}-\psi^{*} R\right) d x \\
\frac{d I_{3}}{d t}=2 P \frac{d x_{0}}{d t}+i \int_{-\infty}^{\infty}\left(2 D\left|\psi_{x}\right|^{2}-\gamma|\psi|^{4}\right) d x+2 i \int_{-\infty}^{\infty}\left(x-x_{0}\right) \\
\times\left(\psi_{x} R^{*}+\psi_{x}^{*} R\right) d x+i \int_{-\infty}^{\infty}\left(\psi R^{*}+\psi^{*} R\right) d x
\end{gathered}
$$

The phase of the pulse evolves according to an additional equation [16]:

$$
\begin{aligned}
\int_{-\infty}^{\infty}\left(\psi \psi_{t}^{*}-\psi_{t} \psi^{*}\right) d x= & i \int_{-\infty}^{\infty}\left(D\left|\psi_{x}\right|^{2}-2 \gamma|\psi|^{4}\right) d x \\
& +i \int_{-\infty}^{\infty}\left(\psi R^{*}+\psi^{*} R\right) d x
\end{aligned}
$$

Equations (8) and (9) are quite general, i.e., they are valid for a large class of NLSE-type evolution equations, including Eq. (1) with arbitrary coefficients. Up to this point, the equations are exact if we use an exact solution of (1) for $\psi$.

In practice, one uses a trial function with a few parameters which depend on $t$. Equations for the evolution of these parameters are found from a system similar to (8). A suitable choice of trial function can be deduced from the general symmetries of the problem, and from results of experiments and numerical simulations. We found that five is the minimum number of parameters needed to describe different localized solutions (see Sec. IV). More parameters may improve the accuracy, but the complexity of the analysis then increases dramatically. Since the number of the moments should correspond to the number of the parameters, we consider only five moments in this work.

The method of moments has been applied successfully to different problems described by the perturbed NLSE $[13,17,18]$. The method was also used for the CGLE in Ref. [19], where a simplified trial function was considered. For special problems, even the first two equations (8) may be sufficient when we deal with exact two-dimensional reductions of the CGLE [20]. In more complicated cases, we need more equations. It happens that the complete set of equations (8) is the minimum required for modeling the pulsating solitons.

\section{TWO MODELS}

The choice of trial function in this work is motivated by numerical simulations of the CGLE (1). It was found $[6,8,9]$ that, for various sets of the system parameters, a dissipative soliton is a single-humped pulse with phase modulation. Here, we consider two forms of the trial function in order to demonstrate that the results vary only slightly, provided a reasonable choice of the function has been made.

\section{A. Sech pulse}

The first type of trial function has the form of a sech function:

$$
\psi(x, t)=A \operatorname{sech}\left(\frac{x-x_{0}}{w}\right) e^{i\left[\phi+b\left(x-x_{0}\right)+c\left(x-x_{0}\right)^{2}\right]},
$$

where $A(t), w(t)$, and $x_{0}(t)$ are the amplitude, width, and position of the pulse maximum, respectively, $\phi(t)$ is the phase shift, $b(t)$ is the linear phase coefficient, and $c(t)$ is the chirp parameter. The phase in Eqs. (10) is expanded up to the second order. This form differs from the trial function used in Ref. [19], where only linear terms in the phase were considered. We emphasize that the chirp is highly important for solutions of the CGLE. As numerical simulations show, even stationary solitons have appreciable phase modulation, not to mention more complicated localized waves, such as pulsating and exploding solitons.

Now, the generalized moments can be expressed in terms of the soliton parameters in the trial function. Evaluation of integrals (6) and (7), with the help of Eq. (10), gives the following expressions:

$$
\begin{gathered}
Q=2 A^{2} w, \quad P=-2 i A^{2} w b, \quad I_{1}=2 A^{2} w x_{0}, \\
I_{2}=\left(\pi^{2} / 6\right) A^{2} w^{3}, \quad I_{3}=i\left(2 \pi^{2} / 3\right) A^{2} w^{3} c .
\end{gathered}
$$

Then, using Eqs. (8), one can obtain a set of ordinary differential equations for the soliton parameters in Eq. (10):

$$
\begin{gathered}
Q_{t}=F_{1} \equiv 2 Q\left[\delta-\beta\left(b^{2}+\frac{1}{3 w^{2}}+\frac{\pi^{2}}{3} c^{2} w^{2}\right)+\frac{\epsilon}{3} \frac{Q}{w}+\frac{2 \mu}{15} \frac{Q^{2}}{w^{2}}\right], \\
w_{t}=F_{2} \equiv 2 D c w+\beta\left(\frac{8}{\pi^{2} w}-\frac{16 \pi^{2}}{15} c^{2} w^{3}\right)-\frac{2 \epsilon}{\pi^{2}} Q-\frac{\mu}{\pi^{2}} \frac{Q^{2}}{w}, \\
c_{t}=F_{3} \equiv 2 D\left(\frac{1}{\pi^{2} w^{4}}-c^{2}\right)-\frac{\gamma}{\pi^{2}} \frac{Q}{w^{3}}-4\left(\frac{1}{3}+\frac{1}{\pi^{2}}\right) \beta \frac{c}{w^{2}} \\
-\frac{8 \nu}{15 \pi^{2}} \frac{Q^{2}}{w^{4}}, \\
x_{0, t}=F_{4} \equiv b\left(D-\frac{2 \pi^{2}}{3} \beta c w^{2}\right), \\
b_{t}=F_{5} \equiv-\frac{4}{3} \beta\left(\frac{1}{w^{2}}+\pi^{2} c^{2} w^{2}\right) b .
\end{gathered}
$$

Equation (9) determines the evolution of the phase shift $\phi$. Since the phase shift $\phi$ does not appear on the right-hand sides of Eqs. (12) explicitly, we ignore this parameter for the rest of the paper.

We make a further reduction of Eqs. (12). We note that, for $\beta>0$, the value of the linear phase $b$ always tends to zero for $t \rightarrow \infty$. Then, the soliton center $x_{0}(t)$ tends to a constant value for $t \rightarrow \infty$. This allows us to consider a system with only three variables, viz., $Q, w$, and $c$. In other words, the three-dimensional subset describes the asymptotic dynamics of the five-dimensional model (12) for $t \rightarrow \infty$. We also men- 
tion that, for $\beta=0$, moving solitons with velocity $x_{0, t}=b D$ are possible. However, such solutions are structurally unstable because they disappear for any small value of $\beta$.

\section{B. Generalized Gaussian pulse}

As numerical simulations show [6,9], pulsating solitons change their shape during one period from a bell-shaped pulse to a flat-top pulse. Therefore, we consider a second trial function which is a combination of Gaussian and superGaussian types of functions:

$$
\psi(x, t)=A \exp \left(-\frac{x^{2}}{w^{2}}-\frac{x^{4}}{4 m w^{4}}+i c x^{2}\right),
$$

where $A(t), w(t)$, and $c(t)$ have the same meaning as in Eq. (10). The constant $m$ can be chosen arbitrarily, but it is independent of $t$. Note that, following the discussion in Sec. IV A, $x_{0}(t)$ and the linear phase $b$ are taken to be zero implicitly in the ansatz. This leaves three parameters $A, w$, and $c$ to be found from the three-dimensional model.

We found that the trial function Eq. (13), with $m>0$, gives better results than the sech function or the Gaussian function alone or the super-Gaussian function with a quartic term alone. Also, we found that the case $4 m=1$ agrees well with the numerical simulations of the CGLE, so that here we consider this case only. However, this value is not critical and other values of $m$ can be used. The general case for arbitrary $m$ is presented in the Appendix.

Since the function (13) is symmetric in $x$, the integrals $P$ and $I_{1}$ are identically zero. Other moments in Eqs. (6) and (7), for $4 m=1$, are given by

$$
\begin{gathered}
Q=1.051 A^{2} w, \quad I_{2}=0.1448 Q w^{2}, \\
I_{3}=4 i c I_{2} .
\end{gathered}
$$

Then Eqs. (8) result in the following dynamical model $(4 m=1)$ :

$$
\begin{aligned}
Q_{t}= & F_{1} \equiv \frac{Q}{w^{2}}\left(2 \delta w^{2}-3.737 \beta-1.158 \beta c^{2} w^{4}+1.433 \epsilon w Q\right. \\
& \left.+1.143 \mu Q^{2}\right), \\
w_{t}= & F_{2} \equiv \frac{1}{w}\left(2.142 \beta+2 c w^{2}-0.8738 \beta c^{2} w^{4}-0.2896 \epsilon w Q\right. \\
& \left.-0.3254 \mu Q^{2}\right), \\
c_{t} & =F_{3} \equiv \frac{1}{w^{4}}\left(6.453-2 c^{2} w^{4}-1.237 w Q-1.319 \nu Q^{2}\right. \\
& \left.-19.62 \beta w^{2} c\right) .
\end{aligned}
$$

As expected, the equations for $Q_{t}, w_{t}$, and $c_{t}$ in (12) and (15) are similar. The only difference lies in the numerical values of the coefficients of the terms. Note also that the definitions of the characteristic lengths in the trial functions are different. The characteristic lengths are related to the full width at half maximum $\left(W_{F W H M}\right)$ of $|\psi|^{2}$ as follows:

$$
W_{F W H M}=2 \cosh ^{-1}(\sqrt{2}) w_{S}=[2(\sqrt{1+4 \ln 2}-1)]^{1 / 2} w_{G G},
$$

so that $w_{S} \approx 0.7788 w_{G G}$, where $w_{S}$ is $w$ in the sech pulse, Eq. (10), while $w_{G G}$ is $w$ in the generalized Gaussian pulse Eq. (13).

\section{ANALYSIS OF THE MODELS}

In this section, we analyze the stationary states and their stability in models (12) and (15). We relate the fixed points and attractors of the two models to various types of localized solutions of the CGLE (1).

Fixed points (FPs) of Eqs. (12) with $x_{0}=b=0$, or of Eqs. (15), are found from the set of algebraic equations $F_{j}=0, j$ $=1,2,3$. The stability of the FPs is determined from the analysis of the eigenvalues $\lambda_{j}, j=1,2,3$, of the Jacobian matrix $M_{i j}=\partial F_{i} / \partial p_{j}$, where $\left\{p_{1}, p_{2}, p_{3}\right\} \equiv\{Q, w, c\}$, and $i$ $=1,2,3$. When the real part of any eigenvalue becomes positive, the corresponding fixed point becomes unstable. Since the characteristic equation for the eigenvalues is cubic, then either $\lambda_{1}=\lambda_{2}^{*}$ and $\lambda_{3}$ is real, or all three $\lambda_{j}$ are real.

Below, we analyze the models (12) with $x_{0}=b=0$ and (15) for different values of the parameters $\nu, \epsilon, \mu$, and $\beta$. We set $\delta=-0.1$ in the numerical examples in the rest of the paper.

\section{A. Plane waves}

The sets of Eqs. (12) or (15) have three formal stationary states at $w \rightarrow \infty, c=0$, and

$$
A_{1}=0, \quad A_{2,3}^{2}=-\frac{5}{8} \frac{\epsilon}{\mu} \pm \sqrt{\left(\frac{5}{8} \frac{\epsilon}{\mu}\right)^{2}-\frac{15}{8} \frac{\delta}{\mu}}
$$

for Eqs. (12), and

$$
A_{1}=0, \quad A_{2,3}^{2}=-0.5964 \frac{\epsilon}{\mu} \pm \sqrt{\left(0.5964 \frac{\epsilon}{\mu}\right)^{2}-1.584 \frac{\delta}{\mu}}
$$

for Eqs. (15). These solutions correspond to plane waves with $k=0$ discussed in Sec. II. The amplitudes in Eqs. (17) and (18) should be compared with exact expressions (4) that gives an accuracy estimate of the method of moments. The solution with the amplitude $A_{2}$ is unstable, while those with $A_{1}$ and $A_{3}$ are stable at least in the plane $c=0$. The existence of the stable states indicates also that there exist regions of attraction to these states at large $w$. We will use this fact in analysis of the stability of localized waves.

\section{B. Localized waves}

Here we consider stationary states with finite width, $\infty$ $>w>0$. First, we compare the results of numerical simulations of the CGLE (1) with those of the models (12) and (15). Then we study attractors of Eqs. (12) in the $(\epsilon, \nu)$ plane for $\beta=0.08$ and $\mu=-0.1$ in more detail. In Sec. VI, we analyze the bifurcation diagrams for different values of $\beta$.

The results of numerical simulations [6] of the CGLE (1) for $\beta=0.08$ and $\mu=-0.1$ are shown in Fig. 1(a). The figure 


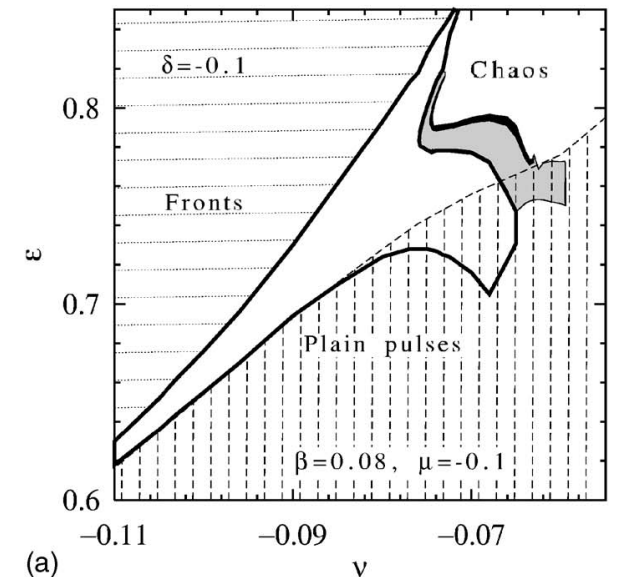

(a)

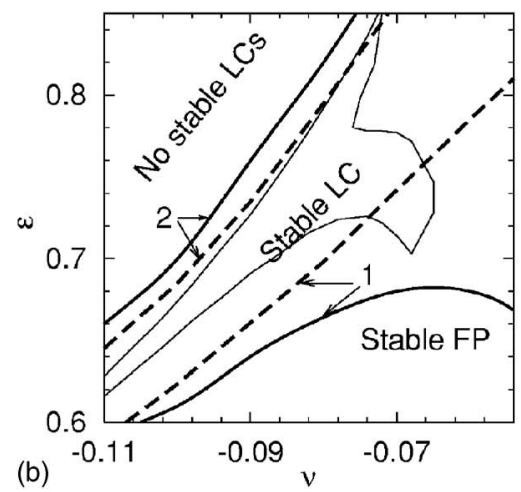

FIG. 1. (a) Regions of existence of various solutions obtained from numerical simulations [6] of the CGLE (1). (b) Regions of existence and stability of FPs and limit cycles of the reduced systems in the $(\nu, \epsilon)$-plane. The region between the two solid (dashed) lines 1 and 2 corresponds to the region of existence of stable LCs in the model Eqs. (12) [ Eqs. (15)]. The region for pulsating solitons is copied from (a) to (b) for comparison. The system parameters are shown in (a).

shows the bifurcation boundaries between various types of localized waves that exist at particular values of the system parameters. The region with vertical shading corresponds to stationary solitons. Solutions describing two fronts moving in opposite directions exist in the region with horizontal shading. Pulsating solitons with periodic or chaotic variations of their parameters occupy the area between these two regions.

To continue the discussion, it is useful to define four types of fixed points for the three-dimensional model:

$$
\begin{gathered}
S_{1}=\left\{(-,+), \lambda_{1}^{\star},(-, 0)\right\}, \\
S_{2}=\{(-, 0),(-, 0),(-, 0)\}, \\
U_{1}=\left\{(+,+), \lambda_{1}^{\star},(-, 0)\right\}, \\
U_{2}=\left\{(-,+), \lambda_{1}^{\star},(+, 0)\right\},
\end{gathered}
$$

where the variables in curly brackets are complex eigenvalues $\left\{\lambda_{1}, \lambda_{2}, \lambda_{3}\right\}$ of the fixed point. The symbols in parentheses show that the corresponding parts of $\lambda_{j}=\left(\operatorname{Re} \lambda_{j}, \operatorname{Im} \lambda_{j}\right)$ are either positive $(+)$, negative $(-)$, or zero. The types $S_{1}$ and $S_{2}\left(U_{1}\right.$ and $\left.U_{2}\right)$ correspond to stable (unstable) FPs.

The bifurcation diagram for the models (12) and (15) is shown in Fig. 1(b). When the value of gain $\epsilon$ is small, there are no FPs in the system. The threshold for FP existence, $\epsilon_{e x}$, can be estimated roughly from Eq. (5) as $\epsilon_{e x} \approx 2 \sqrt{\delta \mu}$. For the parameters in Fig. 1, $\epsilon_{e x} \approx 0.2$. If $\epsilon>\epsilon_{e x}$ then there are two FPs. In the region below the solid (dashed) curve 1 for model (12) [model (15)], one FP is of the type $S_{1}$, while the other FP is of the type $U_{2}$. The second point, $U_{2}$, does not change its type and is unstable in the whole square region shown in Fig. 1(b). Therefore, the bifurcation line 1 in Fig. 1(b) is related to the transformation of the first FP. The amplitude $A$ of the first (second) FP is related to the amplitude $u_{3}\left(u_{2}\right)$ of the stable (unstable) plane wave, and it can be estimated roughly from Eq. (2), taking $k=0$.

Curve 1 is the bifurcation boundary (threshold) where the stable FP of type $S_{1}$ becomes an unstable one of type $U_{1}$. The following condition is satisfied at the threshold:

$$
\operatorname{Re}\left(\lambda_{1}\right)=\operatorname{Re}\left(\lambda_{2}\right)=0 .
$$

The loss of stability of a FP can either be a result of a merging with another unstable FP, or be due to the creation of (annihilation with) a limit cycle (LC) [21]. The latter transition corresponds to a supercritical (subcritical) Hopf bifurcation [21]. Since the number of the FPs does not change in the whole area in Fig. 1(b), the curve 1 is related to the threshold of the Hopf bifurcation in the models (12) and (15).

If the Hopf bifurcation is supercritical, a stable LC would appear exactly at the same value of $\epsilon$ where the FP loses stability. When the Hopf bifurcation is subcritical, the stable LC should appear before the FP becomes unstable [21]. In the latter case, the stable FP and stable LC coexist for a certain interval of the system parameters. Numerical simulations of Eqs. (12) and (15) show that for the set of parameters in Fig. 1(b), the bifurcation is supercritical. The loss of stability of the FP $S_{1}$ is accompanied by the creation of a stable limit cycle.

Close to curve 2 in Fig. 1(b), the period of the LC tends to infinity. There is no stable LC above curve 2. The soliton energy $Q$ and width $w$ in this region increase monotonically with $t$, while the ratio $Q / w$, which is related to the square of the soliton amplitude $A^{2}$ (cf. Sec. V A), remains roughly constant.

The region in Fig. 1(b) surrounded by the thin solid curve corresponds to the area of existence of pulsating solitons of the CGLE, as found from direct numerical simulations. It is copied from Fig. 1(a). Comparison of Figs. 1(a) and 1(b) shows a clear correspondence between the attractors (FPs and LCs) of the two models (12) and (15) and stable localized solutions of the CGLE. Specifically, a FP of the model (12) or (15) corresponds to a stationary soliton, and a LC obtained in the reduced model corresponds to a pulsating soliton. The solution with almost constant $A$ and increasing $w$ [the area above the curve 2 in Fig. 1(b)] approximates two fronts moving in opposite directions. Such a solution was observed in numerical simulations of the CGLE [6]. 
It is obvious that the dynamics of pulses in the CGLE is more complicated than that provided by the models (12) and (15). For example, there is a coexistence of stationary and pulsating pulses, as found in Ref. [6] and presented in Fig. 1(a). However, such a bistability was not found in the models (12) and (15) for the corresponding set of the parameters. Nevertheless, the boundaries obtained in each of the reduced models are fairly close to the exact ones. Thus, each of the models (12) and (15) provide reasonably good qualitative description of the soliton bifurcations inside the squared area of Fig. 1.

In contrast to the stationary state, LCs or pulsating solitons appear due to the dynamical balance between dissipation and energy supply. Pulsations involve periodic variations of the soliton shape parameters $A$ and $w$, and the phase parameter $c$. This behavior is similar to transient dynamics in the integrable NLSE [23], when a pulse adjusts its form to the fundamental soliton via quasiperiodic changes of its amplitude and phase. In the case of the NLSE, such oscillations are damped because the pulse loses energy, radiating linear waves during the transient stage. In the case of pulsating solitons of the CGLE, such oscillations are undamped due to the continuous energy supply.

The parameters of the LC change between curves 1 and 2 . The oscillation period of the LC is finite on curve 1 but it varies along this curve. It is inversely proportional to $\operatorname{Im}\left(\lambda_{1}\right)$ of the first FP. The period increases monotonically with $\epsilon$ at any fixed $\nu$. As mentioned above, the oscillation period of the LC becomes infinite on curve 2, and above it the LC disappears. Examples of limit cycles in three-dimensional phase space for two different sets of the parameters are presented in Fig. 2.

As follows from Fig. 2, the LC becomes elongated in the region of large $w$ when $\epsilon$ increases. However, as was shown in Sec. V A, stable states corresponding to plane waves exist as $w \rightarrow \infty$. Therefore, there should be (separatrix) surfaces that separate the attractive regions of the plane waves from that of the LC. When the size of the LC increases, on increasing the parameter $\epsilon$, the LC can touch the separatrix surface and the LC becomes unstable. Another possible scenario of the LC loss of stability is the period-doubling bifurcation that was found for the CGLE [6] and that exists in the model (12), for example, in the region near $(\epsilon, \nu)$ $=(0.325,0.1)$ and for fixed $(\delta, \beta, \mu)=(-0.1,0.08,-0.1)$.

The dependence of the oscillation period of the LC on $\epsilon$, when other parameters are fixed, is shown in Fig. 3 for each of the reduced models. The curve (solid line), obtained from direct numerical simulations of the CGLE (1), is also shown for comparison. There is an apparent difference in the numerical values of the period due to the drastic reduction in the number of degrees of freedom in the models. However, all three curves have the same qualitative behaviour. In particular, each curve starts with a finite value of the period $T$ at the lower boundary of the region where pulsating solitons exist. The period $T$ increases to infinity when $\epsilon$ reaches the upper boundary. It is clear that the function given by Eq. (13) gives more accurate results.

A comparison of the actual field evolution in $t$ with the one reconstructed from the reduced model is presented in Fig. 4. The results of numerical simulations of the CGLE (1)
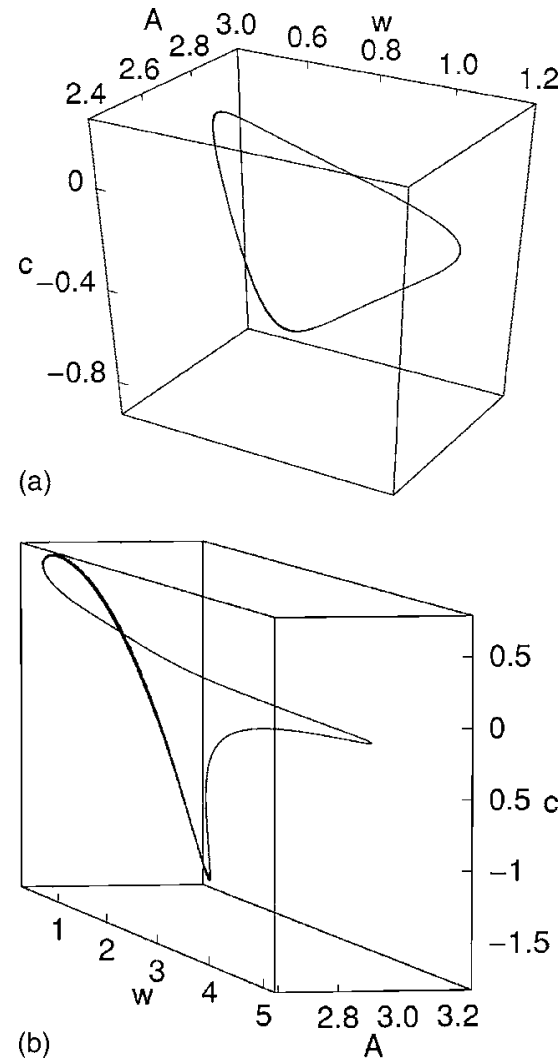

FIG. 2. Examples of limit cycles in $(A, w, c)$ space for the model (12), $\beta=0.08, \mu=-0.1$, and $\nu=-0.09$. $\epsilon=($ a) 0.66 , (b) 0.72 .

are shown in Fig. 4(a). The field, reconstructed from the ansatz (10) and the dynamical systems (12), is plotted in Fig. 4(b). The qualitative features of the dynamics are similar. In particular, the soliton width varies periodically, while the soliton amplitude is close to a constant in each case.

\section{BIFURCATIONS IN THE EXTENDED REGION OF PARAMETERS}

The limited range of parameters in Fig. 1(b) is chosen in order to establish a correspondence with the known numeri-

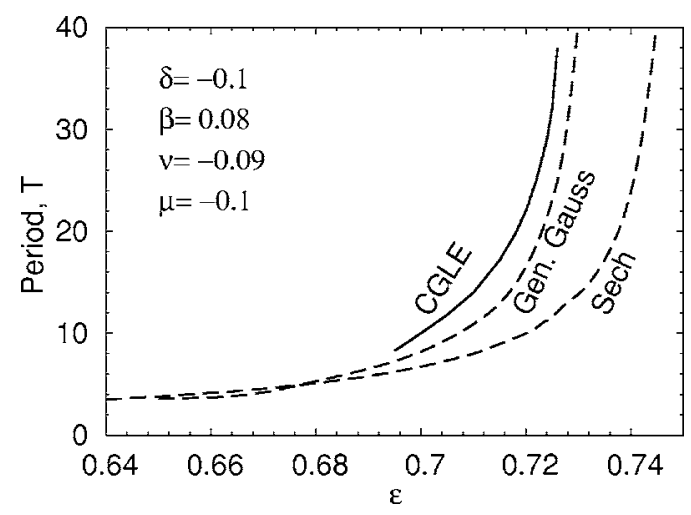

FIG. 3. Period of pulsations $T$ as function of $\epsilon$. The solid line is the result of numerical simulations of the CGLE (1). The dashed lines correspond to the models (12) and (15). 

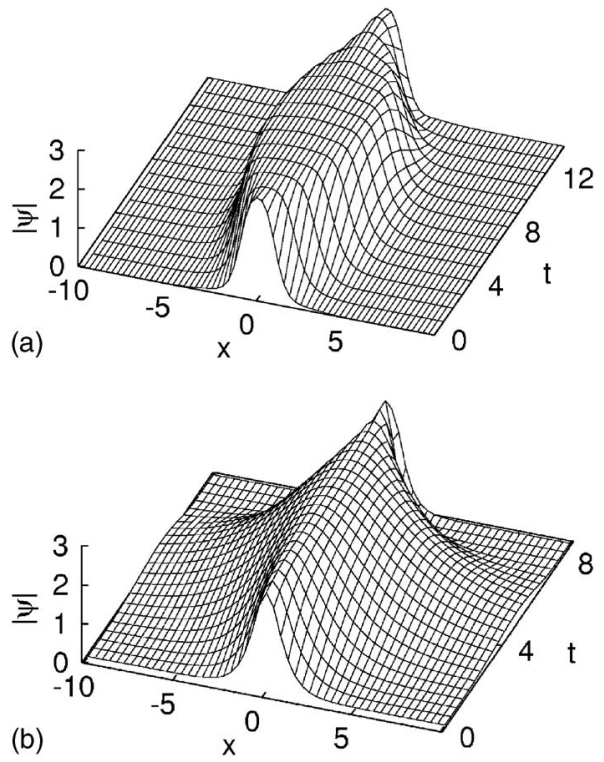

FIG. 4. (a) Pulsating soliton found from numerical simulations [6] of Eq. (1). (b) Soliton reconstructed from the trial function (10) and solution of Eqs. (12). The system parameters are $\beta=0.08, \epsilon$ $=0.66, \mu=-0.1$, and $\nu=-0.1$.

cal results for CGLE. Comparison of the results obtained from Eqs. (12) and (15) with the numerical simulations of the full CGLE justifies the validity of the models. Therefore one can expect that the dynamical systems (12) and (15) can be useful for prediction of the bifurcation thresholds in a wider range of the system parameters. We study one of the reduced models, namely, (12), in a wider range of the parameters to see if there are any other predictions that the simplified dynamical system can provide.

The extended bifurcation diagram for $\beta=0.08$ and $\mu=-0.1$ on the $(\nu, \epsilon)$ plane is shown in Fig. 5. As before, low values of $\epsilon$ cannot support solitons. Thus, there are no FPs in Fig. 5 below the dotted line, which corresponds to $\epsilon_{e x}$ (cf. Sec. V).

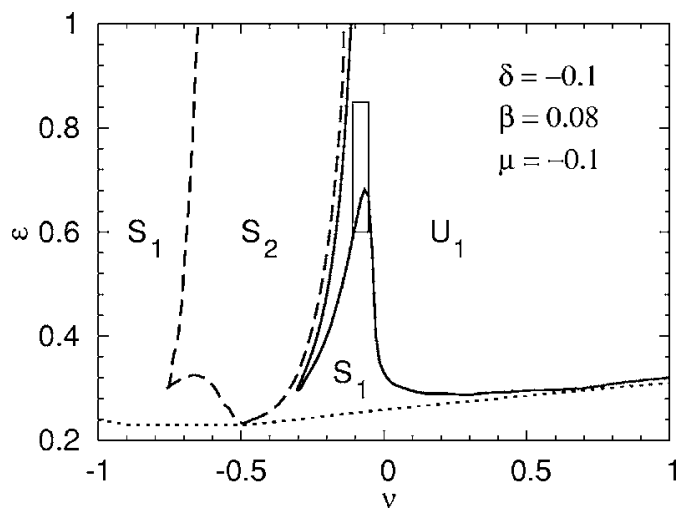

FIG. 5. Bifurcation diagram in the $(\nu, \epsilon)$ plane of the model (12). The solid line separates stable and unstable regions of the first FP. The dashed line separates different stable regions of the first FP. The dotted line is the threshold of existence of the FPs. Symbols correspond to the stability types defined in Eq. (19).

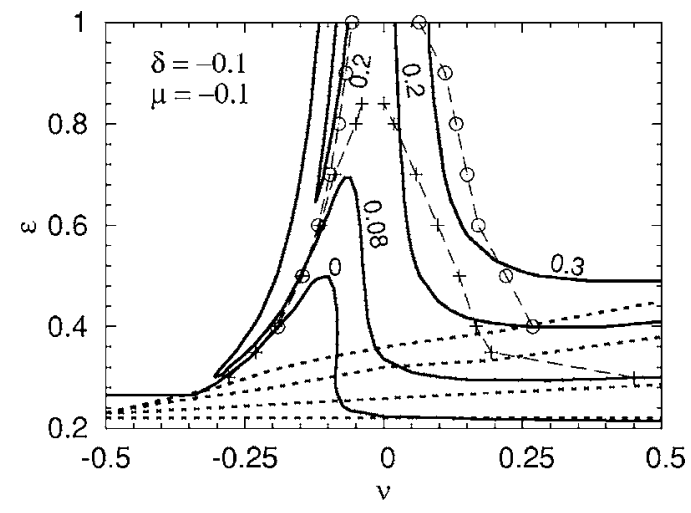

FIG. 6. Bifurcation diagram of the model $(12)$ in the $(\nu, \epsilon)$ plane for $\mu=-0.1$ and different values of $\beta$. The dotted lines are the thresholds of the FP existence. Numbers near solid lines correspond to values of $\beta$. Points correspond to the thresholds found from numerical simulations of the CGLE for $\beta=0.08(+)$ and for $\beta$ $=0.3(\bigcirc)$.

Other curves on the bifurcation diagram in Fig. 5 separate the regions of stability of the first FP. The second FP, of type $U_{2}$, is unstable in the whole area above the dotted line. The symbols in the regions in Fig. 5 show the types of stability of the first FP [see Eq. (19)]. The solid line is a border that separates stable and unstable FPs, while the dashed U-shaped line is a border between stable $S_{1}$ and $S_{2}$ types of the FP. The small rectangle in Fig. 5 shows the limits of parameters taken in Fig. 1. The rectangle is located inside the region of the unstable FP, except for the lower part of it. Therefore, curve 2 and the region of the fronts in Fig. 1(b) are also inside the $U_{1}$ region. We should mention that there is a difference in the extended region between the predictions of the model and results of numerical simulations of the CGLE. We discuss this in detail below.

The transition $S_{1} \rightarrow U_{1}$ across the solid line in Fig. 5 [curve 1 in Fig. 1(b)] corresponds to a Hopf bifurcation. This is an extension of the same bifurcation appearing in Fig. 1(b). Thus, stable LCs should exist everywhere near the solid line in Fig. 5(a). The LC can become unstable or chaotic far from this threshold. However, close to the threshold, the LC is always stable. Also note that there is a narrow vertical stripe with $S_{1}$-type FPs between the two regions with $S_{2}$ - and $U_{1}$-type FPs. Therefore, the transition to a FP of $U_{1}$ type is always from a FP of $S_{1}$ type.

The boundary for the Hopf bifurcation changes when we change the parameter $\beta$ that is shown in Fig. 6. Solid curves represent the Hopf bifurcations for values of $\beta$ ranging from 0 to 0.3. The dotted lines in Fig. 6 are the lower thresholds for the existence of stable solitons. The four dotted lines in Fig. 6 correspond to $\beta=0,0.08,0.2$, and 0.3 , counting from below. As follows from Fig. 6, an increase of the spectral filtering parameter $\beta$ makes wider the region of the stability of the stationary soliton.

The result of numerical simulations of the CGLE is shown by points in Fig. 6. In order to obtain the exact thresholds of the stability, we require massive simulations, involving variation of both the control parameters and initial conditions. Therefore, the following simplified procedure is 
used. The initial condition in all simulations in Fig. 6 was taken in the form $\psi(x, 0)=\operatorname{sech}(x)$, so that $A(0)=w(0)=1$ and $c(0)=0$. The points in Fig. 6 correspond to the threshold when this initial condition is not transformed to the stationary soliton. The region of the stable stationary soliton is between the corresponding curves that agrees qualitatively with the predictions of the model.

As was mentioned above, there are regions in Figs. 5 and 6 where the model (12) gives results which are not fully accurate. The model predicts the existence of stable solitons in the region $\nu \lesssim-0.2$. However, we could not find such states in the numerical simulations of the CGLE. What we observe in that region are exploding solitons, similar to those described in Ref. [22]. An exploding soliton is a long-living soliton that splits suddenly into several pulses. Then they interact with each other, so that the initial form is restored. We note that an exploding soliton looks like a stable stationary state for most of its propagation. Clearly, such behavior cannot be captured by trial functions like (10) or (13). That is why there is a discrepancy in this region. Thus, according to the numerical simulations of the CGLE, the stable stationary solitons exist between the thresholds denoted by points that correspond to the bottom $S_{1}$ area in Figs. 5 and 6.

The results in this section can also be applied to the case $D=\gamma=-1$. The corresponding diagrams are mirror images of Figs. 1, 5, and 6 with the transformation $\nu \rightarrow-\nu$.

Now we make some comments related to the case $D \gamma$ $<0$. Numerical simulations of the CGLE show the existence of stable stationary solitons. However, their forms are different from those given by Eq. (10) or (13). As a consequence, the models (12) and (15) give very rough predictions. Therefore, this case requires a separate study that should be based on a different trial function.

\section{CONCLUSIONS}

Two dynamical models Eqs. (12) and (15) for an approximate description of stationary and pulsating localized solutions of the CGLE, along with the Hopf bifurcation between them, have been considered. The models are constructed using the method of moments. The models describe the time evolution of the main pulse parameters: the pulse amplitude, the width, and the chirp. The models predict qualitatively correct dynamics for a wide range of the system parameters. These forms also provide rough estimates for the bifurcation boundaries between stationary and pulsating solitons.

A correspondence between different types of attractors in the models and various types of localized waves has been demonstrated. Fixed points of the models correspond to stationary solitons, while limit cycles are related to dissipative breathers. It was shown that stable dissipative breathers appear due to the Hopf bifurcation in the system. The models allow one to make a preliminary separation of regions with different types of localized waves over a wide range of the system parameters.

\section{ACKNOWLEDGMENTS}

This work is supported by the Australian Research Council and by the Foundation for Support of Fundamental Stud- ies, Uzbekistan (Grant No. 2F-1-15).

\section{APPENDIX: DEFINITION OF PARAMETERS}

\section{General equations}

Let us consider the generalized nonlinear Schrödinger equation

$$
i \psi_{t}+\frac{D}{2} \psi_{x x}+\gamma|\psi|^{2} \psi=R[\psi],
$$

where $R[\psi]$ accounts for the additional terms in the equation. We assume that a solution of Eq. (A1) has the following form:

$$
\psi(x, t)=A(t) F(y) \exp (i \theta),
$$

where $\quad y=\left[x-x_{0}(t)\right] / w(t) \quad$ and $\quad \theta=\phi(t)+b(t) w(t) y$ $+c(t) w^{2}(t) y^{2}$. If $F(y)$ is a real symmetric function of $y$ then Eqs. (8) result in the following dynamical system for the soliton parameters of the trial function (A2):

$$
\begin{gathered}
Q_{t}=2 A w \int_{-\infty}^{\infty} F \operatorname{Im}(P) d y, \\
w_{t}=2 D c w+\frac{a_{1}}{a_{2}} \frac{A w^{2}}{Q} \int_{-\infty}^{\infty} y^{2} F \operatorname{Im}(P) d y-\frac{A w^{2}}{Q} \int_{-\infty}^{\infty} F \operatorname{Im}(P) d y, \\
c_{t}=\frac{D}{2 a_{2}} \frac{1}{w^{4}} \int_{-\infty}^{\infty}\left(F_{y}\right)^{2} d y-2 D c^{2}-\frac{\gamma}{4 a_{2}} \frac{A^{2}}{w^{2}} \int_{-\infty}^{\infty} F^{4} d y \\
+\frac{1}{2 a_{2} A w^{2}}\left(2 \int_{-\infty}^{\infty} y F_{y} \operatorname{Re}(P) d y+\int_{-\infty}^{\infty} F \operatorname{Re}(P) d y\right), \\
b_{t}=\frac{2 A}{Q} \int_{-\infty}^{\infty} F_{y} \operatorname{Re}(P) d y+\frac{4 A w^{2} c}{Q} \int_{-\infty}^{\infty} y F \operatorname{Im}(P) d y,
\end{gathered}
$$

where $Q=a_{1} A^{2} w, P=R[\psi] \exp (-i \theta)$, and

$$
a_{1}=\int_{-\infty}^{\infty} F^{2} d y, \quad a_{2}=\int_{-\infty}^{\infty} y^{2} F^{2} d y .
$$

A particular case of the system (A3) for $F(y)=\operatorname{sech}(y)$ is presented in Ref. [13].

\section{Parameters for the generalized Gaussian function}

For the trial function (13) with arbitrary $m$, all integrals on the right-hand side of Eqs. (A3) can be written in the form

$$
J_{k, n}(m)=\int_{-\infty}^{\infty} y^{2 k} \exp \left\{-2 n\left[y^{2}+y^{4} /(4 m)\right]\right\} d y,
$$

where $k=[0,4]$ and $n=[1,3]$ are integers. These integrals can be expressed in terms of Bessel, hypergeometric, and 
gamma functions. We define $H(i, j, k)$ to be the hypergeometric function $\left({ }_{1} F_{1}\right)$ with arguments $(i, j, k)$. We also use the modified Bessel function of order $p$ and argument $r, K_{p}(r)$, and the Euler gamma function $\Gamma(r)$. Thus

$$
\begin{aligned}
J_{k, n}(m)= & 2^{-(3 / 4)+k / 2} n^{-(1 / 2)-k}(m n)^{1 / 4+k / 2} \\
& \times\left[\Gamma\left(\frac{1}{4}+\frac{k}{2}\right) H\left(\frac{1}{4}+\frac{k}{2}, \frac{1}{2}, 2 m n\right)-2 \sqrt{2} \sqrt{m n}\right. \\
& \left.\times \Gamma\left(\frac{3}{4}+\frac{k}{2}\right) H\left(\frac{3}{4}+\frac{k}{2}, \frac{3}{2}, 2 m n\right)\right] .
\end{aligned}
$$

We omit lengthy calculations and give here only the final form of the dynamical equations. We also consider some particular cases of fixed $m$. Then integrals $Q$ and $I_{2}$ in the general case of arbitrary $m>0$ can be written as

$$
\begin{gathered}
Q(t)=\sqrt{m} e^{m} K_{1 / 4}(m) A^{2} w, \\
I_{2}(t)=m\left(\frac{K_{3 / 4}(m)}{K_{1 / 4}(m)}-1\right) Q w^{2}, \\
I_{3}(t)=4 i c I_{2} .
\end{gathered}
$$

The equation for $Q_{t}$ has the form

$$
Q_{t}=2 \delta Q+\frac{Q}{s_{1} w^{2}}\left(s_{2} \beta+s_{3} \beta c^{2} w^{4}+\epsilon s_{4} w Q+\mu s_{5} Q^{2}\right),
$$

where

$$
\begin{gathered}
s_{1}(m)=4 m K_{1 / 4}^{3}(m), \\
s_{2}(m)=4 m K_{1 / 4}^{2}(m)\left[K_{1 / 4}(m)-3 K_{3 / 4}(m)\right], \\
s_{3}(m)=2^{11 / 4} e^{-m} m^{5 / 4} K_{1 / 4}^{2}(m)\left[\Gamma\left(-\frac{1}{4}\right) H\left(\frac{3}{4}, \frac{1}{2}, 2 m\right)\right. \\
\left.+2 \sqrt{2 m} \Gamma\left(\frac{1}{4}\right) H\left(\frac{5}{4}, \frac{3}{2}, 2 m\right)\right], \\
s_{4}(m)=8 \sqrt{m} K_{1 / 4}(m) K_{1 / 4}(2 m), \\
s_{5}(m)=8 K_{1 / 4}(3 m) .
\end{gathered}
$$

The trial function can be reduced to a Gaussian by taking $m \rightarrow \infty$. In this case

$$
\begin{aligned}
& \left.\left(s_{2} / s_{1}\right)\right|_{m \rightarrow \infty}=-2,\left.\quad\left(s_{3} / s_{1}\right)\right|_{m \rightarrow \infty}=-2, \\
& \left.\left(s_{4} / s_{1}\right)\right|_{m \rightarrow \infty}=\frac{2}{\sqrt{\pi}},\left.\quad\left(s_{5} / s_{1}\right)\right|_{m \rightarrow \infty}=\frac{4}{\pi \sqrt{3}} .
\end{aligned}
$$
form

The equation for the evolution of the pulse width has the

$$
w_{t}=2 c w+\frac{1}{r_{1} w}\left(r_{2} \beta+r_{3} \beta c^{2} w^{4}+\epsilon r_{4} w Q+\mu r_{5} Q^{2}\right),
$$

$$
\begin{gathered}
r_{1}=24 \sqrt{2} m^{7 / 4} K_{1 / 4}^{3}(m)\left[K_{1 / 4}(m)-K_{3 / 4}(m)\right], \\
r_{2}=6 \sqrt{2} m^{3 / 4} K_{1 / 4}^{2}(m)\left[m r_{2 b} K_{1 / 4}(m)-4(2 m-1)\right. \\
\left.\times(3+m) K_{1 / 4}^{2}(m)+m r_{2 c} K_{3 / 4}(m)\right], \\
r_{3}=24 \sqrt{2} m^{7 / 4} r_{3 c} K_{1 / 4}^{3}(m)+96 e^{-m} 2^{1 / 4} m^{2} r_{3 b} K_{1 / 4}^{2}(m) K_{3 / 4}(m), \\
r_{4}=24 \sqrt{2} m^{5 / 4} K_{1 / 4}(m)\left[K_{1 / 4}(2 m) K_{3 / 4}(m)-K_{1 / 4}(m) K_{3 / 4}(2 m)\right], \\
r_{5}=24 \sqrt{2} m^{3 / 4}\left[K_{1 / 4}(3 m) K_{3 / 4}(m)-K_{1 / 4}(m) K_{3 / 4}(3 m)\right] .
\end{gathered}
$$

Here

$$
\begin{gathered}
r_{2 b}=2(5-28 m) K_{3 / 4}(m)+2(26 m-11) K_{5 / 4}(m)+8 m K_{7 / 4}(m), \\
r_{2 c}=4 m K_{7 / 4}(m)-12 K_{3 / 4}(m), \\
r_{3 b}=2 \sqrt{2} \sqrt{m} \Gamma\left(\frac{5}{4}\right) H\left(\frac{5}{4}, \frac{3}{2}, 2 m\right)-\Gamma\left(\frac{3}{4}\right) H\left(\frac{3}{4}, \frac{1}{2}, 2 m\right), \\
r_{3 c}=4(1+m) K_{1 / 4}(m)-4 m K_{5 / 4}(m) . \quad \text { (A12) }
\end{gathered}
$$

For the Gaussian trial function, the coefficients are found to be

$$
\begin{gathered}
\left.\left(r_{2} / r_{1}\right)\right|_{m \rightarrow \infty}=2,\left.\quad\left(r_{3} / r_{1}\right)\right|_{m \rightarrow \infty}=-2, \\
\left.\left(r_{4} / r_{1}\right)\right|_{m \rightarrow \infty}=-\frac{1}{2 \sqrt{\pi}},\left.\quad\left(r_{5} / r_{1}\right)\right|_{m \rightarrow \infty}=-\frac{4}{3 \pi \sqrt{3}} .
\end{gathered}
$$

The equation for $c_{t}$ is written as

$$
c_{t}=-2 c^{2}-\frac{1}{v_{1} w^{4}}\left(v_{2}+v_{3} w Q+\nu v_{4} Q^{2}-\beta v_{5} c w^{2}\right),
$$

where

$$
\begin{gathered}
v_{1}=48 m^{2} K_{1 / 4}^{2}(m)\left[K_{1 / 4}(m)-K_{3 / 4}(m)\right], \\
v_{2}=-12 m K_{1 / 4}^{2}(m)\left[K_{1 / 4}(m)-3 K_{3 / 4}(m)\right], \\
v_{3}=-12 \sqrt{m} K_{1 / 4}(m) K_{1 / 4}(2 m), \\
v_{4}=-16 K_{1 / 4}(3 m), \\
v_{5}=48 m K_{1 / 4}^{2}(m)\left\{(8 m-1) K_{1 / 4}(m)\right. \\
\left.+2 m\left[5 K_{5 / 4}(m)-9 K_{3 / 4}(m)\right]\right\} .
\end{gathered}
$$

where 
We obtain the Gaussian trial function results for $c_{t}$ by taking the limits $m \rightarrow \infty$.

$$
\left.\left(v_{2} / v_{1}\right)\right|_{m \rightarrow \infty}=-2,\left.\quad\left(v_{3} / v_{1}\right)\right|_{m \rightarrow \infty}=\frac{1}{\sqrt{\pi}},
$$

Equations (A8), (A10), and (A13) for $m=1 / 4$ are reduced to the system (15).

$$
\left.\left(v_{4} / v_{1}\right)\right|_{m \rightarrow \infty}=\frac{8}{3 \pi \sqrt{3}},\left.\quad\left(v_{5} / v_{1}\right)\right|_{m \rightarrow \infty}=-8 .
$$

[1] W. van Saarlos and P. C. Hohenberg, Physica D 56, 303 (1992).

[2] M. C. Gross and P. C. Hohenberg, Rev. Mod. Phys. 65, 851 (1993).

[3] I. S. Aranson and L. Kramer, Rev. Mod. Phys. 74, 99 (2002).

[4] See, e.g., N. Akhmediev and A. Ankiewicz, Solitons: Nonlinear Pulses and Beams (Chapman \& Hall, London, 1997); G. P. Agrawal, Nonlinear Fiber Optics (Academic Press, London, 1989).

[5] R. J. Deissler and H. R. Brand, Phys. Rev. Lett. 72, 478 (1994).

[6] N. Akhmediev, J. M. Soto-Crespo, and G. Town, Phys. Rev. E 63, 056602 (2001).

[7] H. Sakaguchi and B. A. Malomed, Physica D 154, 229 (2001).

[8] J. M. Soto-Crespo, M. Grapinet, Ph. Grelu, and N. Akhmediev, Phys. Rev. E 70, 066612 (2004).

[9] N. Akhmediev and A. Ankiewicz, in Dissipative Solitons, (Springer, Heidelberg, 2005).

[10] E. P. Ippen, Appl. Phys. B: Lasers Opt. 58, 159 (1994).

[11] Z. Bakonyi, D. Michaelis, U. Peschel, G. Onishchukov, and F. Lederer, J. Opt. Soc. Am. B 19, 487 (2002).

[12] P. Kolodner, Phys. Rev. Lett. 66, 1165 (1991).
[13] A. I. Maimistov, Zh. Eksp. Teor. Fiz. 104, 3620 (1993) [JETP 77, 727 (1993)].

[14] E. N. Tsoy and N. Akhmediev, Phys. Lett. A 343, 417 (2005).

[15] J. M. Soto-Crespo, N. Akhmediev and G. Town, J. Opt. Soc. Am. B 19, 234 (2002).

[16] Sh. Watanabe, M. Miyakawa, and N. Yajima, J. Phys. Soc. Jpn. 46, 1653 (1979).

[17] E. N. Tsoy and C. M. de Sterke, Phys. Rev. E 62, 2882 (2000).

[18] F. Kh. Abdullaev, D. V. Navotny, and B. B. Baizakov, Physica D 192, 83 (2004).

[19] M. N. Zhuravlev and N. V. Ostrovskaya, Zh. Eksp. Teor. Fiz. 126, 483 (2004) [JETP 99, 427 (2004)].

[20] N. Akhmediev, A. Ankiewicz, and J. M. Soto-Crespo, J. Opt. Soc. Am. B 15, 515 (1998).

[21] J. Guckenheimer and P. Holmes, Nonlinear Oscillations, Dynamical Systems and Bifurcations of Vector Fields (SpringerVerlag, New York, 1983).

[22] J. M. Soto-Crespo, N. Akhmediev, and A. Ankiewicz, Phys. Rev. Lett. 85, 2937 (2000).

[23] J. Satsuma and N. Yajima, Suppl. Prog. Theor. Phys. 55, 284 (1974). 